\title{
Endovascular Treatment of Wide Necked Ruptured Saccular Aneurysms with Flow-Diverter Stent
}

\author{
Enes DUMAN¹, Ilker COVEN², Erkan YILDIRIM', Cem YILMAZ², H.Ulas PINAR ${ }^{3}$ \\ ${ }^{1}$ Baskent University, Medical Faculty, Konya Research Center, Department of Radiology, Konya, Turkey \\ ${ }^{2}$ Baskent University, Medical Faculty, Ankara Research Center, Department of Neurosurgery, Ankara, Turkey \\ ${ }^{3}$ Baskent University, Medical Faculty, Konya Research Center, Department of Anesthesiology, Konya, Turkey
}

\section{ABSTRACT}

\begin{abstract}
AIM: Flow diverter (FD) stents have been used in the treatment of unruptured intracranial aneurysms. There are a few studies that report the use of these devices in ruptured blister-like aneurysms. We present 5 consecutive patients, who had ruptured intracranial wide necked or side branch close to the neck of saccular aneurysms, with no other treatment options, treated with FD stents and coil embolization.
\end{abstract}

MATERIAL and METHODS: Between September 2012 and April 2015, 139 ruptured aneurysms of 133 consequent patients were treated. Of these, 48 were surgically treated aneurysms. Five of the remaining 85 aneurysms treated with FD stents. Three aneurysms were in the posterior communicating artery, and 2 were in the supraclinoid internal carotid artery (ICA). Partial coil embolization was performed in addition to FD stents in three patients. All patients were treated in the first 3 days after bleeding.

RESULTS: Technical success was $100 \%$. Inappropriate deployment of silk stent and partial thrombus formation occurred in one patient due to the jailed micro-catheter. Inappropriate apposition of stent was corrected with a balloon, and the thrombus resolved with tirofiban, tissue plasminogen activator (t-PA) injections. No other complication or death occurred related to the procedure. One patient who had a giant ICA aneurysm and Fisher grade 4 bleeding died due to vasospasm, cerebral edema and sepsis on the postoperative $13^{\text {th }}$ day. The other patients were followed-up uneventfully with computed tomography angiography (CTA) at $6^{\text {th }}$ month and digital subtraction angiography (DSA) at $12^{\text {th }}$ month.

CONCLUSION: FD stents can be used in the treatment of ruptured large wide necked or side branch close to the neck of saccular aneurysms when other treatment options can not be used.

KEYWORDS: Aneurysm, Intracranial, Endovascular treatment, Flow diverter devices, Subarachnoid hemorrhage

\section{INTRODUCTION}

$\mathrm{S}$ ince 2007, intracranial flow diverter (FD) stents have been used in clinical practice for treatment of intracranial aneurysms $(9,12,14)$. FD stents provides rapid progression of thrombosis within the aneurysm sac by reducing hemodynamic exchange between the aneurysm and parent artery, reducing the risk of aneurysm rupture, and decreasing mass effects related to aneurysm pulsatility (3). Endovascular coil embolization of large and wide neck aneu- rysms with or without remodeling methods (stent or balloon) is associated with low rates (35\%) of initial angiographic occlusion and high rates $(50 \%)$ of recurrence $(4,10,11)$.

Although, there are many reports about use of FD stents in the treatment of ruptured blister-like or dissecting intracranial aneurysms $(2,6,8,10,13)$, to our knowledge these devices have not been used in the treatment of recently ruptured large wide neck or saccular aneurysms that are not suitable to treat with conventional methods. 
Here, we present 5 consecutive patients who had ruptured intracranial large wide neck or side branch close to the neck of saccular aneurysms and were treated with silk FD stents and partial coil embolization as there were no other endovascular or surgical treatment options. The aim was to evaluate the safety and feasibility of FD stent in the treatment of such recently ruptured aneurysms.

\section{MATERIAL and METHODS}

Between September 2012 and April 2015, 139 ruptured aneurysms of 133 consequent patients were treated. Informed consent was obtained from all patients. Of these, 48 were surgically treated aneurysms. Endovascular treatment of 85 aneurysms consisted of selective coiling in 40 aneurysms (47.05\%), stent assisted coiling in 22 aneurysms (25.88\%), balloon assisted coiling in 8 aneurysms (9.41\%), 1 aneurysm $(1.17 \%)$ with intra-aneurysmal flow diverting device (web device) and FD stents in 14 aneurysms (16.47\%). Thirteen patients with 14 aneurysms were treated with FD stents. Of these, 4 were dissecting vertebral or basilar artery aneurysms. Three of them were ruptured blister-like internal carotid artery (ICA) aneurysms. These 7 patients were excluded from the study. The remaining 7 patients had ruptured saccular wide necked aneurysms. Of these, 2 were treated in a staged manner. First, partial selective coil embolization to the aneurysm dome was performed to prevent recurrent bleeding, and FD

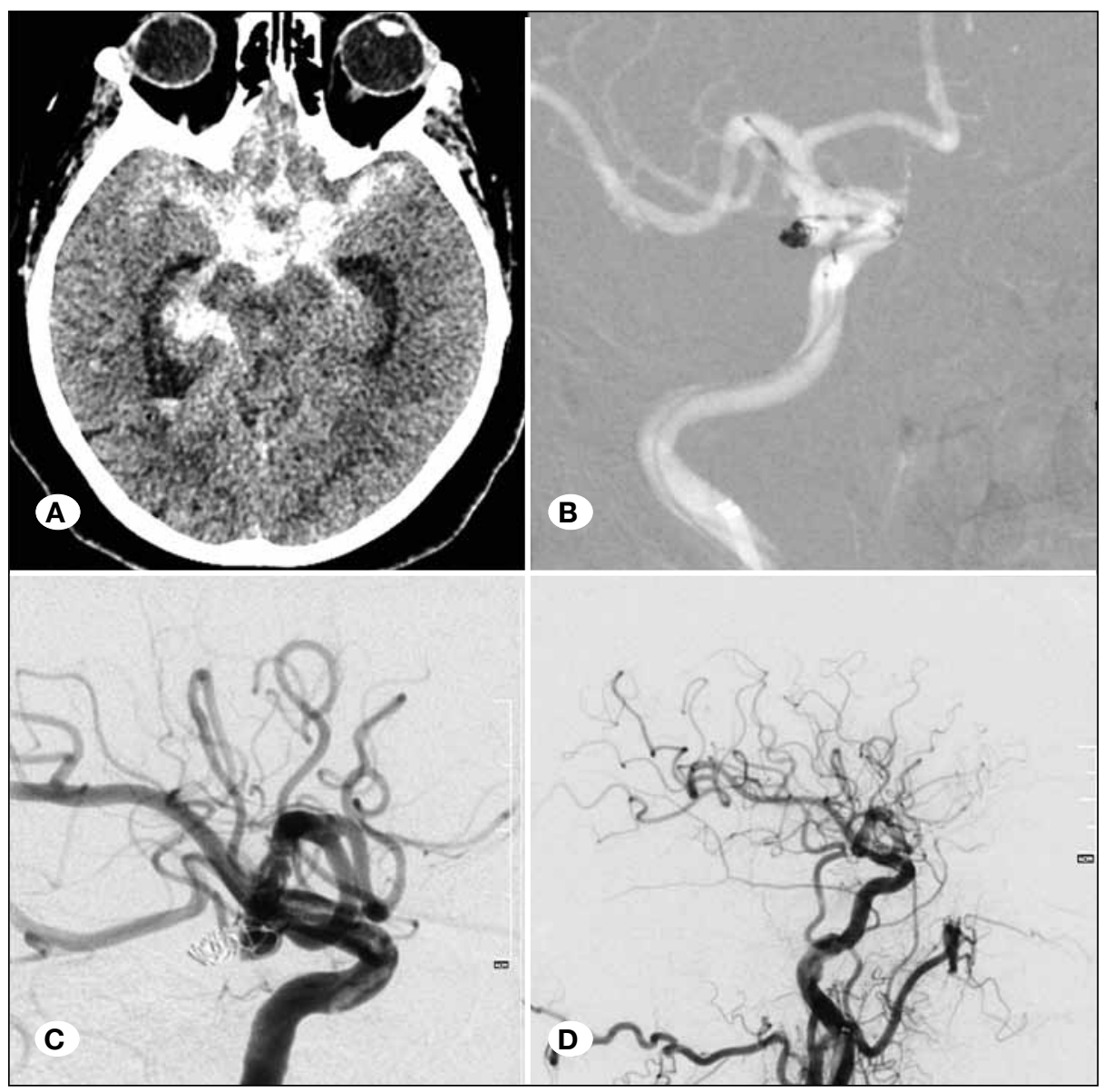

stents were deployed 4-6 weeks after the initial procedure. These 2 patients were also excluded from the study. According to the patients' decisions endovascular treatment protocols were applied instead of surgery. The remaining 5 patients ( 3 female and 2 male) were treated with FD stents in the acute setting. There was absence of $\mathrm{P} 1$ segment of posterior cerebral artery in 2 patients who had posterior communicating artery (P-com) aneurysms (Figures 1A-D, 3A-D), so preservation of the posterior communicating artery was crucial (Figure 3A-D). Total exclusion of the aneurysms from the circulation was not possible without occluding the posterior communicating artery with the usage of remodeling balloons and conventional bare stents. First of all we decided to perform staged treatment, and we tried to embolize the dome of the aneurysm with balloon assisted coiling to prevent recurrent bleeding in the acute phase but it was not possible due to the aneurysm shape. We did not prefer the standard embolization techniques for the other patient who had a p-com aneurysm because of similar reasons. In another patient, the anterior choroidal artery origin was very close to the aneurysm neck and the carotid artery segment was dysplastic. The other two patients had supraclinoid huge wide necked aneurysms (Figures 2A, B; 4A-D). One of them had been treated with coil embolization 5 year ago. He has recurrent amorphous aneurysm that originated from the primary aneurysm neck, and a dysplastic ICA segment (Figure 4A-D).
Figure 1: A 64-year-old female patient. A) Axial CT image shows Fisher grade 3 SAH. B) Road map image after deployment of Silk stent and during partial coiling of right P-Com aneurysm. C) The control angiography shows acute thrombus formation on stent struts and complete occlusion of aneurysm nipple. D) The control angiography shows nearly total resolution of thrombus after selective injection of tirofiban and tPA. 

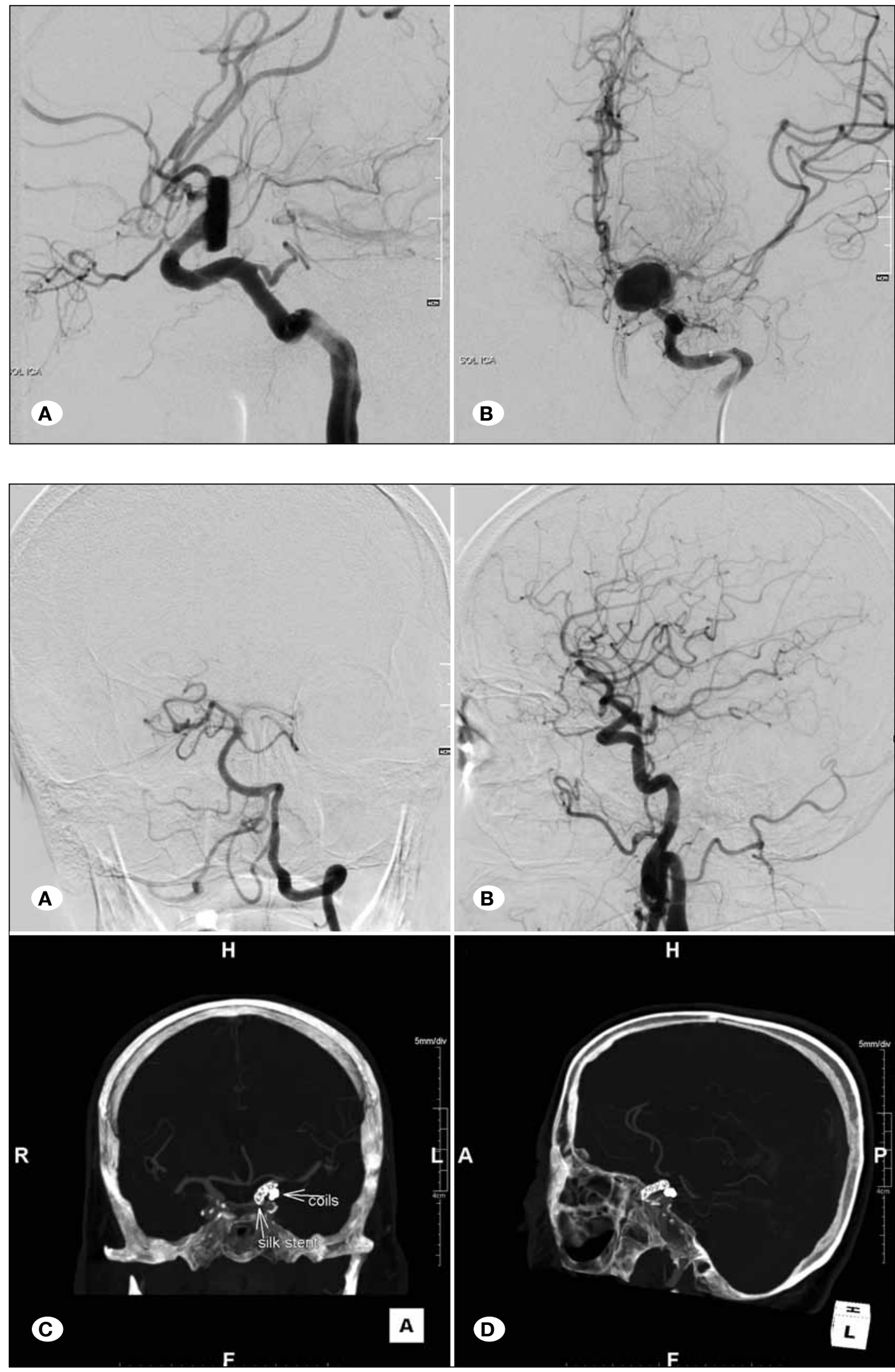

Figure 2: A 47-year-old female patient.

A) Lateral, B) Anteroposterior DSA image shows left giant supraclinoid ICA aneurysm.
Figure 3: A 68-year-old female patient.

A) AP left vertebral artery angiography shows absence of left P1 segment of posterior cerebral artery.

B) Lateral DSA image shows fetal origin of left posterior cerebral artery and ruptured saccular left P-Com aneurysm.

C, D) AP and lateral CTA shows coils and silk stent. 
Partial coil embolization performed in three patients additional to FD stents. The age range was $43-72$ years. Three of the 5 aneurysms were $p$-com saccular aneurysm, and two were supra-clinoid ICA saccular aneurysms. The demographic and clinical characteristics of the patients and morphologic features of the aneurysms are summarized in Table I.

Diagnosis of all aneurysms was done with digital subtraction angiography (DSA) (Artis zee, Siemens, Germany). All patients were treated in the first 3 days after bleeding. All procedures were performed under general anesthesia through the femoral approach. $600 \mathrm{mg}$ of clopidogrel was administered through a nasogastric tube at least 6 hours before the procedure. In vitro platelet function tests for clopidogrel clinical response were not used. An external ventricular drainage catheter was placed only in one patient before initiation of dual antiplatelet therapy. Systemic anticoagulation was provided with IV administration of $5000 \mathrm{IU}$ of heparin (Vasparin $囚$, Mefar, Istanbul, Turkey) after femoral access was obtained. A 6 Fr long vascular sheath (Epsylar, Optimed, Ettlingen, Germany) was introduced to the common carotid artery and a 6 Fr guiding catheter (Fargomax, Balt Extrusion, Montmorency, France) was inserted coaxially to the petrous part of the ICA. $2 \mathrm{mg}$ (10 ml solution) of nimodipine (Nimotop ${ }^{\circledR}$, Bayer Pharma AG, Leverkusen, Germany) was injected over the sheath before guiding catheter placement to prevent catheter-related vasospasm. Aneurysms were jailed before Silk stent deployment in three patients for loosely packed coil embolization. One of the other 2 patients who had saccular aneurysms had a recurrent aneurysm that had been coiled 5 years ago, and the other one had a giant aneurysm. Additional IV heparin infusion was started to achieve a targeted activated clotting time of 250 300 second for at least 12 hours. The patients were followedup with computed tomography angiography (CTA) (Aquilion, Toshiba, Japan) at the $6^{\text {th }}$ month and DSA at the $12^{\text {th }}$ month.

\section{RESULTS}

Five patients ( 3 female and 2 male, with a mean age of 61.4 years) were treated with a silk flow diverter stent in the acute setting of subarachnoid hemorrhage. Three of the aneurysms were $p$-com aneurysms and 2 were supraclinoid ICA aneurysms. The mean aneurysm diameter was $10.32 \mathrm{~mm}$ (range 5.2-20 mm), and the mean dome to neck ratio was 0.88 (range $0.94-0.77$ ). The technical success rate was $100 \%$. Inappropriate deployment of silk stent occurred in one patient due to the

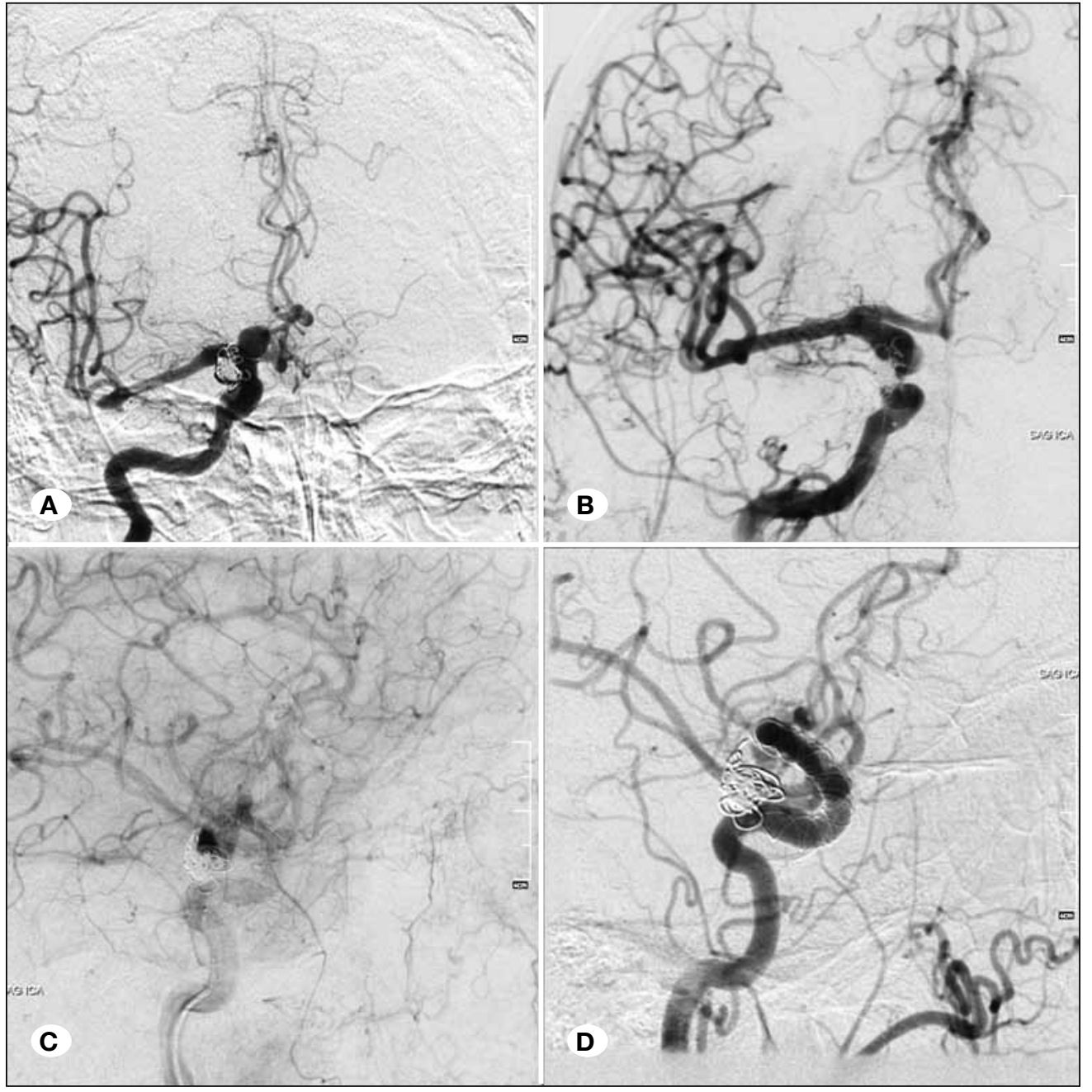

Figure 4: A 72-year-old male patient. A, B) AP right ICA injection shows recurrent amorphous aneurysm, C, D) right anterior oblique images show stagnation of flow in aneurysmal sac and patent distal ICA branches. 
jailed microcatheter. The probable reason was termination of the proximal part of the stent at a curved ICA segment where the jailed microcatheter was positioned on the opposite wall of the ICA. Such a position of the microcatheter prevents wall apposition of the stent during deployment. Inappropriate apposition of the stent was corrected with a re-modeling balIoon (Eclipse, Balt Extrusion, and Montmorency, France). After balloon remodeling, thrombus formations on stent struts was seen on follow-up angiography. Immediate injection of $4 \mathrm{mg}$ tirofiban $\mathrm{HCl}$ (Aggrastat ${ }^{\circledR}$, DSM Pharmaceuticals, North Carolina, ABD), and $10 \mathrm{mg}$ of tPA (Actilyse ${ }^{\circledR}$, Boehringer Ingelheim Pharma, Riss, Germany) resolved the thrombus completely. 1 $\mathrm{mg} / \mathrm{h}$ IV tirofiban infusion was continued for 24 hours (Figure 1A-D). All patients were kept on $75 \mathrm{mg}$ clopidogrel and 300 $\mathrm{mg}$ of coraspin a day for one year. No other minor or major (bleeding or thromboembolism) complication or death related to the procedure occurred. A female patient who had a giant ICA aneurysm and Fisher grade 4 bleeding died due to vasospasm, cerebral edema and sepsis on the postoperative $13^{\text {th }}$ day (Figure 2A, B). The other four patients are under follow up uneventfully. The clinical status of the patients at discharge, follow-up and complications are summarized in Table II.

\section{DISCUSSION}

FD stents are increasingly being used in the endovascular treatment of intracranial aneurysms. FD stents provide rapid progression of thrombosis within the aneurysm sac, reduce the risk of aneurysm rupture, and decrease mass effects related to aneurysm pulsatility (3). The majority of patients have large neck aneurysms or recurrent aneurysms. However, precise indications for the use of FD stents are still uncertain. Although the majority of reported studies in the literature include patients who have no other endovascular or surgical options, two new reports about the use of FD stents in ruptured aneurysms have recently been published. The reports consist of ruptured blister aneurysms of supraclinoid ICA. Aydın et al. reported treatment of eleven ruptured blister aneurysms of supraclinoid ICA with a Silk FD stent (2), Cinar et al. reported treatment of seven ruptured blister aneurysms of supraclinoid ICA with a Pipeline FD stent (5). In our study, Silk FD stent deployment was performed in five patients with ruptured large wide necked or side branch close to the neck of saccular aneurysms. To our knowledge, Berge et al. reported the largest series of Silk stent deployment. They treated 77 patients, including un-ruptured aneurysms with a 5-year cumulated risk of hemorrhage of $14.5 \%$ following the ISUIA11 criteria (30/77 aneurysms), symptomatic carotid cavernous (29/77) and carotid ophthalmic (8/77) aneurysms, and previously ruptured aneurysm or multiple aneurysms on a dysplastic carotid segment (10/77 aneurysms) (3). The major concerns about the use Silk stents in ruptured aneurysms are the necessity of anti-aggregant therapy and poor apposition. Berge et al. indicate 9 poor appositions of 63 stents (12.3\%) in their study (3). This situation also occurred in one of our patients due to a jailed micro catheter, and the problem was solved by use of a remodeling balloon. We think that the possible causes of poor apposition of Silk stents are either 1) the stent has poor radial force, or 2) multiple backward and forward manipulations are needed during deployment.

Table I: The Demographic and Clinical Characteristics of Patients and Morphologic Features of Aneurysms

\begin{tabular}{cccccccccc}
\hline Patients & Age & Sex & Location & Type & Size & D/N Ratio & $\begin{array}{c}\text { Hunt and } \\
\text { Hess }\end{array}$ & $\begin{array}{c}\text { Fisher } \\
\text { Coma Score }\end{array}$ \\
\hline 1 & 47 & F & L. Supraclinoid ICA & Saccular & $20 \times 15 \mathrm{~mm}$ & 0.91 & 5 & 4 & 7 \\
\hline 2 & 64 & F & Right P-com & Saccular & $6.8 \times 4.7 \mathrm{~mm}$ & 0.77 & 3 & 3 & 11 \\
\hline 3 & 72 & M & R. Supraclinoid ICA & Saccular & $14 \times 7 \mathrm{~mm}$ & 0.89 & 1 & 2 & 15 \\
\hline 4 & 68 & F & Left P-com & Saccular & $5.6 \times 3.3 \mathrm{~mm}$ & 0.91 & 2 & 3 & 15 \\
\hline 5 & 56 & M & Left P-com & Saccular & $5.2 \times 3.9 \mathrm{~mm}$ & 0.94 & 3 & 3 \\
\hline
\end{tabular}

*Glasgow coma score at admission, D/N: Dome/Neck, F: Female, M: Male.

Table II: The Clinical Status of the Patients at Discharge, Follow up and Complications

\begin{tabular}{ccccccc}
\hline Patients & ${ }^{*}$ Glasgow & ${ }^{* *}$ MRS & Complication & Follow up time & Status & Cause of death \\
\hline 1 & 3 & 6 & - & - & died & Vasospasm+sepsis \\
\hline 2 & 15 & 1 & thrombus & 4 months & alive & - \\
\hline 3 & 15 & 1 & - & 14 months & alive & - \\
\hline 4 & 15 & 1 & - & 12 months & alive & - \\
\hline 5 & 15 & 1 & - & 17 months & alive & - \\
\hline
\end{tabular}

*Glasgow coma score at discharge. ${ }^{* *}$ MRS: Modified Rankin Scale. 
Another important point about the use of FD stents in bleeding aneurysm is that these devices can not provide immediate aneurysm thrombosis. In their study, Berge et al. reported only 5 of 76 aneurysms ( $7 \%$ ) showed complete stasis after the Silk stent procedure (3). We decided to combine partial coil embolization with FD stents to overcome such a devastating result in ruptured aneurysms. Partial coil embolization was performed in 3 patients after deploying the FD stent. One patient had a recurrent aneurysm that had been coiled 5 years ago and one had a giant aneurysm. Subsequent stasis was achieved after deployment of the FD stent in the giant aneurysm.

The use of anti-aggregant therapy and regimen in the treatment of ruptured aneurysms is not well established. We preferred to perform procedures under systemic anticoagulation, and patients were loaded with dual anti-platelet therapy with aspirin and clopidogrel at least 6 hour before the intervention. Additionally we continued systemic anticoagulation with IV heparin infusion $500 \mathrm{IU} /$ hour for at least 12 hours after the procedure, because per-oral clopidogrel loading achieves effective platelet inhibition within approximately 6-8 hours (1, 7). Only in one patient (20\%) was acute thrombus formation seen during the procedure, and this problem was resolved with the use of tirofiban and t-PA. We did not avoid the use of these drugs as the aneurysm was completely excluded from the circulation with the coils and FD stents.

There may be a major concern about the usage of FD stents in the treatment of such patients. Why did we not prefer the other treatment options such as balloon remodeling coiling or stent assisted coiling? There was absence of the P1 segment of the posterior cerebral artery in 2 patients who has $p$-com aneurysms, so preservation of the posterior communicant artery was crucial (Figure 3A-D). Total exclusion of aneurysms from the circulation was not possible without occluding the posterior communicating artery with usage of remodeling balloons and conventional bare stents. First of all we decided to perform staged treatment, and we tried to embolize the dome of aneurysm with balloon assisted coiling to prevent recurrent bleeding in the acute phase. However, it was not possible due to the aneurysm shape. We did not prefer the standard embolization techniques for the other patient who had a p-com aneurysm because of similar reasons. The anterior choroidal artery origin was very close to the aneurysm neck and the carotid artery segment was dysplastic. The other two patients had supraclinoid huge wide necked aneurysms. One of them had been treated with coil embolization 5 year ago. He had a recurrent amorphous aneurysm that originated from the primary aneurysm neck, and a dysplastic ICA segment (Figure 4A-D).

The major limitation of the study is the small number of patients.

\section{- CONCLUSION}

FD stents can be used in the treatment of ruptured large wide necked or side branch close to the neck of saccular aneurysms when other treatment options can not be performed. If possible, staged endovascular treatment with coils and FD stents may be safer option in the treatment of such recently ruptured aneurysms. We need further clinical studies with large series about the use of FD stents in such patients.

\section{REFERENCES}

1. Altenburg A, Haage P: Antiplatelet and anticoagulant drugs in interventional radiology. Cardiovasc Intervent Radiol 35: 30-42, 2012

2. Aydin K, Arat A, Sencer S, Hakyemez B, Barburoglu M, Sencer A, İzi N: Treatment of blood blister-like aneurysms with flow diverter Silk stent. J Neurointerv Surg 7: 202-209, 2015

3. Berge J, Biondi A, Machi P, Brunel H, Pierot L, Gabrillargues J, Kadziolka K, Barreau X, Dousset V, Bonafé A: Flow-diverter silk stent for the treatment of intracranial aneurysms: 1-year follow-up in a multicenter study. AJNR Am J Neuroradiol 33: 1150-1155, 2012

4. Campi A, Ramzi N, Molyneux AJ, Summers PE, Kerr RS, Sneade M, Yarnold JA, Rischmiller J, Byrne JV: Retreatment of ruptured cerebral aneurysms in patients randomized by coiling or clipping in the International Subarachnoid aneurysm Trial (ISAT). Stroke 38: 1538-1544, 2007

5. Cinar C, Oran I, Bozkaya H, Ozgiray E: Endovascular treatment of ruptured blister-like aneurysms with special reference to the flow-diverting strategy. Clin Neurol Neurosurg 115:920-925, 2013

6. Consoli A, Nappini S, Renieri L, Limbucci N, Ricciardi F, Mangiafico S: Treatment of two blood blister-like aneurysms with flow diverter stenting. J Neurointerv Surg 4: e4, 2012

7. Katsaridis V, Papagiannaki C, Violaris C: Embolization of acutely ruptured and unruptured wide-necked cerebral aneurysms using the Neuroform 2 stent without pretreatment with antiplatelets: A single center experience. AJNR Am J Neuroradiol 27: 1123-1128, 2006

8. Kulcsár Z, Wetzel SG, Augsburger L, Gruber A, Wanke I, Rüfenacht DA: Effect of flow diversion treatment on very small ruptured aneurysms. Neurosurgery 67:789-793, 2012

9. Lylyk P, Miranda C, Ceratto R, Ferrario A, Scrivano E, Luna HR, Berez AL, Tran Q, Nelson PK, Fiorella D: Curative endovascular reconstruction of cerebral aneurysms with the Pipeline embolization device: The Buenos Aires experience. Neurosurgery 64: 632-642, 2009

10. Molyneux AJ: Indications for treatment of cerebral aneurysms from an endovascular perspective: The creation of an evidence base for interventional techniques. Neurosurg Clin N Am 16: 313-316, 2005

11. Molyneux AJ, Kerr RS, Yu LM, Clarke M, Sneade M, Yarnold JA, Sandercock P: International subarachnoid aneurysm trial (ISAT) of neurosurgical clipping versus endovascular coiling in 2143 patients with ruptured intracranial aneurysms: A randomized comparison of effects on survival, dependency, seizures, rebleeding subgroups and aneurysm occlusion. Lancet 366: 809-817, 2005

12. Nelson PK, Lylyk P, Szikora I, Wetzel SG, Wanke I, Fiorella D: The Pipeline embolization device for the intracranial treatment of aneurysms trial. Am J Neuroradiol 32: 34-40, 2011

13. Rasskazoff S, Silvaggio J, Brouwer PA, Kaufmann A, Nistor A, lancu D: Endovascular treatment of a ruptured blood blisterlike aneurysm with a flow diverting stent. Interv Neuroradiol 16: 255-258, 2010

14. Szikora I, Berentei Z, Kulcsar Z, Marosfoi M, Vajda ZS, Lee W, Berez A, Nelson PK: Treatment of intracranial aneurysms by functional reconstruction of the parent artery: The Budapest experience with the Pipeline embolization device. Am J Neuroradiol 31:1139-1147, 2010 